\title{
Revisando los trabajos prácticos experimentales en la enseñanza universitaria
}

\author{
LORENZO, María G. 1
}

\author{
Filiaciones institucionales \\ ${ }^{1}$ Universidad de Buenos Aires, Facultad de Farmacia y Bioquímica. Centro de Investigación y Apoyo a la Edu- \\ cación Científica. \\ Educación Científica Consejo Nacional de Investigaciones Científicas y Técnicas (CONICET), Argentina.
}

\section{Correspondencia}

glorenzoffyb@gmail.com

\section{Resumen}

La enseñanza de las ciencias experimentales ha estado ligada al desarrollo del trabajo experimental desde siempre. Sin embargo, la planificación y el diseño de las actividades prácticas no han recibido la misma atención que otros aspectos, como las teorías o la evaluación, al pensar en los diseños curriculares de las asignaturas. Consecuentemente, en este artículo se revisan algunas características generales de los trabajos prácticos de los laboratorios de ciencias experimentales, a la vez que se proponen algunos criterios para su revisión y análisis. Se presentan los trabajos prácticos abiertos como alternativa a las actividades experimentales más tradicionales.

\section{Palabras clave}

enseñanza de las ciencias | clases de laboratorio | prácticas experimentales 


\section{Summary}

Experimental sciences teaching and bench laboratory work have been linked for the very beginning. However, there were not the same attention on the practical activities planning and design than others, as theoretical models or assessment, in order to organize the subject-matters of the course of studies. In this paper, some general characteristics of experimental work for science teaching are consequently reviewed. Some several analysis criteria are also proposed. Open practical activities are presented as an alternative as traditional teaching practices.

\section{Keywords}

science teaching / laboratory classes / experimental practices

\section{Los trabajos prácticos de laboratorio en la enseñanza de las ciencias experimentales}

Las carreras universitarias vinculadas a las ciencias experimentales y a las ciencias de la salud se caracterizan por incluir en la gran mayoría de sus asignaturas lo que suele denominarse trabajo práctico experimental o trabajo práctico de laboratorio como un componente esencial de las actividades de enseñanza obligatorias para el estudiantado. De acuerdo con la asignatura, estos trabajos prácticos pueden variar entre realizar un proceso de destilación o una titulación en una clase de química, medir el pasaje de los rayos de luz a través de diferentes tipos de lentes o construir un circuito eléctrico en clases experimentales de física, observar diferentes preparados de tejidos animales y vegetales sobre la platina de un microscopio en el área de la biología, o sembrar bacterias en una placa de Petri en el área de la microbiología. $Y$ así podríamos seguir enumerando una gran cantidad de actividades que, si bien son diferentes, tienen algunas características en común. Brevemente, podemos describirlas como actividades donde los estudiantes deben realizar algún tipo de tarea que excede la actividad intelectual para comprometer al cuerpo. Estas actividades experimentales, además de fundamentarse en modelos teóricos ineludibles, apuntan a desarrollar destrezas y habilidades de manipulación de instrumentos y objetos (motricidad fina), de observación, de registro, de comunicación (Jalii, 2006).

Podemos referirnos entonces a dos grandes tipos de procedimientos que tienen lugar durante una clase práctica experimental (Lorenzo, Reverdito, Perillo, y Salerno, 2001):

a) Los procedimientos intelectuales (PI), que a su vez pueden agruparse en aquellos que permiten a los estudiantes reconocer un determinado objeto 
o suceso (PIR) y aquellos que se ponen en juego a la hora de supervisar una acción y tomar decisiones sobre alguna situación para ejercer cierto grado de control sobre la misma (PIC), siendo estos últimos de una mayor exigencia cognitiva que los primeros.

b) Los procedimientos sensoriomotores (PS) que involucran las acciones vinculadas a la motricidad fina con el objetivo de modificar el sistema (PSA) y aquellos relacionados con la especialización de los sentidos que permiten transformar hechos en datos de una observación (PSO). Por ejemplo, reconocer el punto de una titulación, la aparición de un precipitado en una solución o registrar la temperatura en un termómetro.

Además, este tipo de clases, también promueve cierta manera de hacer las cosas, de comportarse dentro de ese espacio, que hace a las actitudes propias del ejercicio profesional hacia donde se orienta la enseñanza (Lorenzo, 2018).

Queda claro entonces que las clases prácticas de laboratorio son un escenario complejo en donde se desarrolla una parte importante de la enseñanza de ciencias (López Rua y Tamayo Alzate, 2012, Rodríguez y Hernández, 2015, Sánchez et al., 2019) que se incluyen en los programas académicos con el fin de que los estudiantes adquieran competencias y herramientas propias del quehacer científico y sus implicancias (Hofstein y Mamlok-Naaman, 2007). Sin embargo, esta misma complejidad conlleva ciertas dificultades en el cumplimiento de estos propósitos (Hodson, 1994, Hofstein, 2017, Lorenzo, Reverdito, Blanco y Salerno, 2012).

Por ello, en este artículo nos proponemos presentar y discutir algunos criterios que consideramos útiles para revisar los trabajos prácticos experimentales que se realizan en los laboratorios de ciencias naturales a la luz de algunos aportes de la literatura propia de la didáctica de las ciencias. De modo de ofrecer herramientas a los profesores de ciencias para revisitar sus propias prácticas, este trabajo se organiza en torno a los siguientes objetivos:

- Revisar las características de las actividades experimentales de laboratorio y sus propósitos.

- Presentar consideraciones para la planificación, el diseño y la evaluación de actividades experimentales.

- Comentar algunas tendencias actuales sobre los trabajos prácticos de tipo abierto. 


\section{Los trabajos prácticos de laboratorio en la literatura especializada}

La enseñanza formal y sistemática de las ciencias experimentales puede reconocer sus orígenes en el siglo XIX y, desde entonces, ha recurrido a los trabajos de laboratorio como parte indiscutible de sus actividades curriculares (Blosser, 1988, Nakhleh, Polles y Malina, 2002). Las primeras investigaciones en el campo de una incipiente didáctica de las ciencias se enfocaron en las ventajas y limitaciones en el uso del laboratorio para la enseñanza de las ciencias (Blosser, 1983), muchas de estas últimas relacionadas con el costo de los recursos materiales y la demanda de tiempo que implica el trabajo experimental (Insausti, 1997, Insausti y Merino, 2000).

Un primer compendio de objetivos que deberían cumplirse durante las clases experimentales fueron propuestos por Shulman y Tamir en el Second Handboook of Research on Teaching (Travers, 1973:1119), muchos de los cuales continúan vigentes hasta nuestros días, aunque resulta difícil reconocer la especificidad del laboratorio en estas metas:

- Desarrollar habilidades manuales, inquisitivas, de investigación, organizativas y comunicativas.

- Favorecer el aprendizaje de conceptos (por ejemplo, hipótesis, modelos teóricos, categorías taxonómicas).

- Promover actividades cognitivas (pensamiento crítico, resolución de problemas, aplicación, análisis, síntesis, entre otras).

- Facilitar la comprensión de la naturaleza de la ciencia y de la empresa científica, el trabajo de los científicos, la existencia de múltiples métodos científicos, las relaciones entre la ciencia y la tecnología y entre diversas ciencias entre sí.

- Estimular actitudes como curiosidad, interés, objetividad, confianza, perseverancia, tomar riesgos, satisfacción, responsabilidad, consenso y colaboración.

Por otra parte, otros investigadores han planteado otros propósitos a la hora de pensar en la realización de los trabajos prácticos de laboratorio:

Como un gran primer objetivo, Gupta (2001) considera que el laboratorio debe servir para la enseñanza de los diferentes métodos y técnicas que se utilizan en la actividad experimental, tales como familiarizarse con el equipamiento de los laboratorios, hacer mediciones, observaciones, registrar datos, planear experimentos, etc., que corresponderían a los procedimientos intelectuales y sensoriomotores mencionados más arriba. Aunque también advierte que estas 
actividades deben presentarse a los estudiantes de un modo desafiante y no como una tarea mecánica de baja demanda cognitiva.

- Un segundo objetivo ampliamente perseguido en la enseñanza de las ciencias, y al que se le atribuye gran parte del fracaso de este tipo de prácticas, es la utilización de la actividad experimental como recurso para ilustrar, ejemplificar o complementar las clases de tipo teóricas, lo que Seré (2002) denomina «la práctica al servicio de la teoría», que provoca una «visión distorsionada» del trabajo científico (Reigosa Castro y Jiménez Aleixandre, 2000).

- También se reconoce al trabajo en el laboratorio un tercer objetivo vinculado a metas afectivas (Johnstone y Al-Shuaili, 2001) que hacen referencia a las actitudes. Por un lado, aquellas actitudes hacia la ciencia, como el interés, la motivación y la satisfacción, y por otro, las actitudes científicas, como el pensamiento independiente y crítico, la objetividad, la responsabilidad, entre otras.

A partir de los 2000, las publicaciones desarrollan una nueva forma de concebir los trabajos prácticos de laboratorio atendiendo fundamentalmente a los cambios socioculturales acaecidos y a las nuevas formas de entender el mundo, como por ejemplo el cuidado y el respeto por la salud humana, por la vida y el ambiente, las nuevas demandas laborales y profesionales y los cambios en el perfil estudiantil (Byers, 2002, Seré, 2002). Si bien inicialmente los estudios se focalizaron en las propuestas de enseñanza en laboratorio e innovaciones para el nivel secundario (Franco Moreno, Velasco Vásquez y Riveros Toro, 2017), en los últimos tiempos la didáctica de las ciencias se encuentra repensando sus estructuras y prácticas para el nivel universitario (Lorenzo, 2017, Seery, Agustian, y Zhang, 2018).

En este sentido, lo que en un principio se pensó como una oportunidad para entrar en contacto con los fenómenos naturales, se plantea ahora al laboratorio como un entorno multidimensional en donde, a partir de las interacciones entre estudiantes y profesores, pueda construirse nuevo conocimiento mediado por la utilización de diversos instrumentos científicos cada vez más sofisticados. El laboratorio es un entorno rico en información (Domin, 1999) que condiciona no solo lo que se aprende o debe aprenderse sino también el modo en que se aprende.

En resumen, el trabajo en el laboratorio debería entrenar a los alumnos para el uso de técnicas de manera eficaz y segura en un proceso (largo) que incluya la ayuda y el soporte de los docentes en la realización de las actividades prácticas que podrían denominarse «demostraciones» (Glasson, 1989, Hofstein y Lunetta, 1982) y otras actividades que requieran que cada estudiante haga con sus propias manos ciertas tareas que involucren su cuerpo y su mente hasta adquirir confianza y seguridad en sí mismo para desenvolverse adecuadamente. Y que al mismo tiempo 
le permitan desarrollar la especialización de sus sentidos, anticipar el fenómeno, comprenderlo y relacionar los niveles macroscópico y submicroscópico. Para ello, las prácticas experimentales deberían convertirse en un espacio donde el error, la prueba, la superación del fracaso formen parte de los contenidos de aprendizaje.

\section{Las actividades experimentales en la enseñanza}

Si bien a lo largo de los últimos años ha habido muchos intentos de modificar los trabajos prácticos de laboratorio y existen propuestas puntuales de innovación, una gran parte de las actividades experimentales continúa presentando un diseño de «recetas» y/o «mostraciones», lo que limita las posibilidades de aprendizaje de los estudiantes.

Por otro lado, persiste la creencia de que el contacto directo con los fenómenos naturales que ocurren en el laboratorio es suficiente para aprender, para comprender los conceptos y para resolver los problemas profesionales a futuro. De igual manera, esta creencia tiene su contrapartida en la enseñanza al considerar que el trabajo en el laboratorio de investigación se traduce linealmente en buenas prácticas docentes.

Esta idea sobre la importancia de meter las manos en la masa entra en tensión con las nuevas formas de la «experimentación» que utilizan dispositivos informáticos, tanto en los ámbitos de investigación científica (como, por ejemplo, los programas de modelado molecular o docking) como en las actividades de enseñanza en entornos virtuales o en los laboratorios remotos (aquellos donde se usa Internet, webcams y otros dispositivos que pueden manipularse a la distancia para obtener datos) (Calvo, Zulueta, Gangoiti y López, 2008), de los cuales se cuenta con varios años de experiencia en su implementación (Matarrita y Concari, 2018).

Por último, vale la pena replantearse al propio espacio del laboratorio como escenario para la enseñanza y el aprendizaje. Las múltiples variables que deben tenerse en cuenta a la hora de desenvolverse en un laboratorio, desde la vestimenta, las medidas de seguridad, hasta los distintos materiales e instrumentos, en su mayoría desconocidos para los estudiantes, generan un entorno con un exceso de información que podría saturar la memoria de trabajo de los estudiantes, en especial de aquellos que dan sus primeros pasos en estos ambientes. 


\section{Evaluación y diseño de actividades experimentales}

En primer lugar, si el objetivo es revisar las propuestas de trabajo práctico experimental que efectuamos en nuestras asignaturas para mejorarlos es importante disponer de un conjunto de criterios diferentes que permita analizarlas o evaluarlas. Seguramente, muchas de las actividades prácticas propuestas para la enseñanza en el laboratorio fueron pensadas por su relevancia, los perfiles profesionales de su época y porque posibilitaban desarrollar en los estudiantes destrezas y habilidades necesarias para su formación. No obstante, suele ocurrir que dichas actividades se repitan sin que haya una revisión o actualización para adecuarlas a las nuevas necesidades. Es por ello que, en este apartado, presentaremos algunas ideas y criterios para repensar los trabajos prácticos vigentes, así como para planificar un nuevo diseño para las actividades experimentales.

Antes de avanzar, daremos algunas definiciones para evitar confusiones al referirnos a estos conceptos (Reverdito y Lorenzo, 2007):

Un experimento incluye un conjunto de actividades, acciones o situaciones en las que el resultado es, además de hipotético, incierto. Los experimentos son los que suelen llevarse a cabo en las situaciones «reales» de la práctica científica, donde el investigador planifica la actividad sobre la base de su conocimiento controlando determinadas variables y a la espera de un resultado que no siempre cumple con sus expectativas. No es común que en una actividad de enseñanza se incluyan verdaderos experimentos.

En cambio, una actividad experimental hace referencia a un conjunto de actividades, acciones o situaciones propuestas por los docentes para su enseñanza en la que el resultado, aunque es desconocido (y hasta sorprendente) para los estudiantes, está predeterminado por una teoría consensuada científicamente, planificado didácticamente y cuyo objetivo primordial es que los estudiantes aprendan algún contenido disciplinar seleccionado de modo intencional, que puede ser algún concepto, técnica, destreza, actitud o una combinación de ellos.

\section{Un marco teórico para pensar las prácticas en el laboratorio}

La planificación de las actividades prácticas experimentales, necesariamente intencional, requiere de ciertos fundamentos teóricos que la sustenten. Un modelo que aporta un marco de referencia interesante para pensar sobre los trabajos 
prácticos es el presentado en el Esquema 1, conocido como el triángulo de Alex Johnstone $(1993,2000)$. Si bien fue propuesto inicialmente para la química, puede extenderse al análisis de otras disciplinas. En él, Johnstone propone un triángulo en el que interactúan tres niveles representacionales diferentes o de diferentes aspectos de un cierto constructo disciplinar que han de ser tenidos en cuenta para su genuina comprensión.

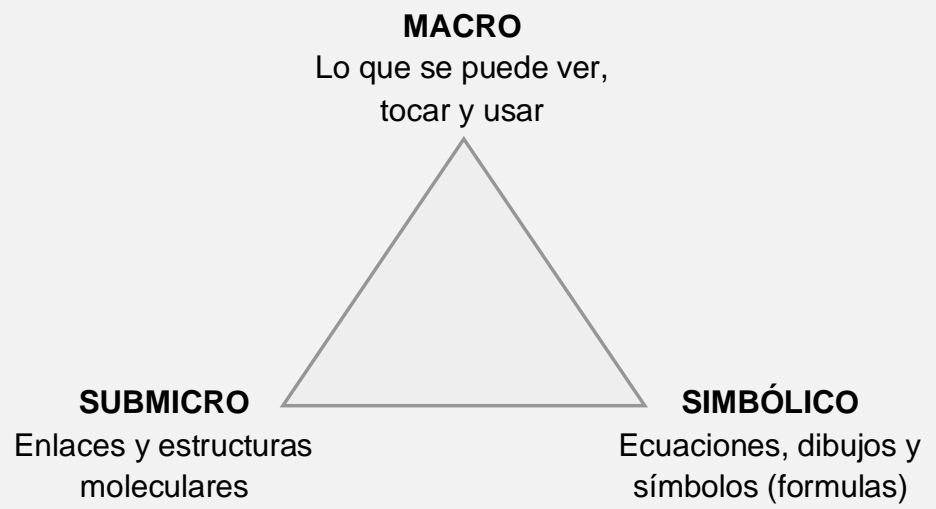

Esquema 1. Modelo de los niveles representacionales de la ciencia (adaptado de Johnstone, 1993)

Los niveles macro y submicro hacen referencia a cuestiones del tamaño de los objetos de estudio de una disciplina. En el caso de la química, se asume que el nivel macro corresponde a los fenómenos y transformaciones que pueden ser percibidos por los sentidos a través de la experiencia; mientras que el nivel submicroscópico corresponde al mundo invisible de átomos y moléculas. Y, para poder hablar sobre y comunicarse entre científicos, fue necesaria la creación de un lenguaje simbólico compuesto por distintos sistemas externos de representación (Treagust, Chittleborough y Mamiala, 2003) que completan el tercer vértice del triángulo de Johnstone. Según este autor, un profesor (o un científico) se ubicaría en el centro del triángulo y podría «saltar» de un vértice a otro sin dificultad. Es decir que, por ejemplo, al ver la decoloración de una solución magenta por el agregado de agua oxigenada en un medio ligeramente acidulado, un químico puede plantear las fórmulas químicas y las ecuaciones que explican a nivel atómico y electrónico lo ocurrido, o viceversa. En tanto que para un estudiante novato estos aspectos resultarían aislados e inconexos. Este modelo no es inequívoco (Lorenzo, 2008, Talanquer, 2011), sin embargo, tiene gran utilidad pedagógica para repensar las prácticas de enseñanza. En este sentido, podríamos preguntarnos a qué nivel o niveles de pensamiento químico refieren las actividades propuestas en nuestros trabajos prácticos de laboratorio o si se promueve de manera efectiva la 
interconexión entre dichos niveles, entre otras cuestiones a tener en cuenta a la hora de evaluar una propuesta de trabajo en el laboratorio.

\section{Criterios para el análisis de un trabajo práctico}

A continuación, enunciaremos algunas consideraciones que contribuyen a la evaluación o al diseño a la hora de planificar la realización de un trabajo práctico. Para una mayor claridad expositiva, las organizamos respetando los elementos que componen el triángulo didáctico: la enseñanza, la disciplina y los aprendizajes que promueven.

\section{Desde el punto de vista de su enseñanza}

- El tiempo necesario para su implementación, ¿es adecuado a las condiciones y planificación curriculares? Aunque puede pasar que nos quedemos cortos con la propuesta de actividades y el trabajo práctico se termine antes de lo previsto, la mayoría de las veces somos demasiado ambiciosos y recargamos de actividades una sesión de laboratorio; por lo tanto, no damos tiempo suficiente para avanzar con detalle y precisión en el paso a paso, no hay oportunidades para corregir errores o repetir alguna actividad.

- Los recursos materiales, ¿son fáciles de conseguir a un costo razonable? Hay que conocer el presupuesto disponible en relación con la cantidad de estudiantes y la propuesta de actividades. ¿Se están planeando actividades para que los alumnos realicen de manera individual? ¿En parejas? ¿Grupales? ¿Cuál es el equipamiento disponible en el laboratorio? ¿Qué y cuánto hay que comprar? ¿Dónde se compran los materiales y los reactivos? ¿Se dispone de un lugar para almacenar adecuadamente los materiales y los reactivos? ¿Se requieren equipos especiales? ¿Se consiguen los repuestos de los equipos?

- Las instalaciones disponibles, ¿son adecuadas para la realización de las actividades propuestas? ¿Se dispone de mobiliario apropiado? ¿Cuántas personas pueden habitar el espacio simultáneamente sin correr riesgos? ¿Se dispone de ventilación, buena iluminación, piletas, correcto descarte de residuos?

- ¿Están previstas las medidas de seguridad necesarias? ¿Se cumple con las normas de seguridad de la institución? ¿Está habilitado el laboratorio para realizar ese tipo particular de actividades experimentales? ¿Cuenta 
con matafuegos (en condiciones)? ¿Los teléfonos de emergencias están fácilmente accesibles? ¿Existe un protocolo de descarte de materiales peligrosos? ¿Y ante un caso de emergencia?

- El número de docentes, ¿permite mantener una buena relación (numérica) docente/alumno que posibilite a su vez la tutorización adecuada y minimice posibles riesgos?

2. Desde el punto de vista de la disciplina

- ¿Qué contenidos disciplinares incluye el trabajo práctico? ¿Son relevantes esos contenidos en el contexto de la disciplina? ¿A qué criterios responde la selección de contenidos? ¿Están adecuadamente secuenciados? (Lorenzo, 2018).

- Transferencia de contenidos: ¿son transferibles a otros trabajos prácticos de la misma asignatura? ¿A otras asignaturas? ¿A la práctica profesional?

- Evaluación de contenidos: ¿se incluyen los contenidos del trabajo práctico en las evaluaciones de la asignatura? Ya sea afirmativo o negativo, ¿qué implicaciones tiene esto sobre el trabajo práctico?

\section{Desde el punto de vista de los aprendizajes que promueve}

- Los estudiantes, ¿disponen de los conocimientos previos necesarios para la realización del trabajo práctico? ¿Disponen de las competencias básicas para realizar las actividades experimentales propuestas?

- Las actividades a realizar, ¿promueven aprendizajes significativos? ¿permiten construir conceptos o son meras repeticiones mecánicas?

- ¿Promueven la adquisición de autonomía?

- ¿Favorecen el desarrollo de destrezas generales/específicas?

- ¿Ayudan a desarrollar actitudes científicas/trabajo en equipo/otras?

- ¿Cómo y en qué momento se evalúan los aprendizajes construidos durante el trabajo práctico?

- Y finalmente, si un alumno no pudiera concurrir al TP, ¿de qué se perdería? 


\section{Consideraciones para el diseño de un trabajo práctico experimental}

A pesar de que ya han pasado muchos años, la propuesta de Gil Pérez y Valdés Castro (1996) resume una serie de consideraciones que siguen vigentes a la hora de diseñar un trabajo práctico. Las transcribiremos con muy pocas modificaciones seguidamente:

1. Presentar situaciones problemáticas abiertas de un nivel de dificultad adecuado al desarrollo cognitivo de los estudiantes que permitan la toma de decisiones y favorezcan la reflexión sobre la relevancia de aquéllas para promover su interés y dar sentido a su estudio en el contexto de las interacciones entre la ciencia, la tecnología y la sociedad (CTS) (Lorenzo y Farré, 2016), para evitar un estudio descontextualizado y socialmente neutro.

2. Potenciar los análisis cualitativos, significativos, que ayuden a comprender y a delimitar las situaciones planteadas, de modo de poder formular preguntas operativas sobre lo que se busca para evitar caer en la falsa creencia sobre el papel esencial de las matemáticas como instrumento de investigación.

3. Plantear la emisión de hipótesis como actividad fundamental de la investigación científica a partir de marcos teóricos consensuados, susceptible de orientar el tratamiento de las situaciones y de hacer explícitas las ideas y conocimientos de los estudiantes sobre el tópico. Operativizar cuidadosamente las hipótesis considerando la derivación de consecuencias contrastables y prestando la debida atención al control de variables.

4. Dar un mayor grado de libertad a los estudiantes para que puedan elaborar sus propios diseños y para planificar la actividad experimental a partir de ciertos criterios generales y con el material disponible, que debería ir incorporando nuevos dispositivos, medidas de seguridad y control a medida que estén disponibles. 
5. Plantear el análisis detenido de los resultados (su interpretación física, fiabilidad, posibles errores), con base en el cuerpo de conocimientos disponible, de las hipótesis manejadas y de los resultados de «otros investigadores» (otros equipos de estudiantes o artículos científicos). Favorecer, a la luz de los resultados, las necesarias revisiones de los diseños, de las hipótesis e, incluso, del planteamiento del problema. Prestar una particular atención, en su caso, a los conflictos entre los resultados y las concepciones iniciales, y facilitar, de una forma funcional, los cambios conceptuales. Dedicar tiempo suficiente al tratamiento de los datos experimentales, a su organización y tabulación, las diferentes formas de graficación así como la interpretación de la información gráfica. (Idoyaga, Moya y Lorenzo, 2017)

6. Plantear la consideración de posibles perspectivas (replanteamiento del estudio a otro nivel de complejidad, problemas derivados...) y contemplar, en particular, las implicaciones CTS del estudio realizado (aplicaciones, repercusiones negativas, entre otras).

7. Pedir un esfuerzo de integración que considere la contribución del estudio realizado a la construcción de un cuerpo coherente de conocimientos, así como las posibles implicaciones en otros campos de conocimientos.

8. Conceder una especial importancia a la elaboración de memorias científicas que reflejen el trabajo efectuado y puedan servir de base para resaltar el papel de la comunicación y el debate en la actividad científica. La escritura es una actividad central del trabajo científico, y en el caso del laboratorio cobran singular interés los informes de laboratorio, como, por ejemplo, el que se presenta en la Tabla 1. Otro formato que actualmente contribuye tanto a la presentación de resultados como al debate colectivo de los mismos son las láminas (pósteres) o presentaciones en Power Point $\circledast$ o Prezzi, que son ampliamente utilizados en los congresos científicos y cada vez más como actividades de enseñanza en contextos educativos. 


\section{INFORME DE TRABAJO REALIZADO}

\begin{tabular}{|c|c|}
\hline OBJETIVO & ¿Qué se quería hacer? \\
\hline FUNDAMENTACIÓN & $\begin{array}{l}\text { ¿Cuál es el problema que se intenta investigar? ¿Por qué se está ha- } \\
\text { ciendo este experimento? } \\
\text { HIPÓTESIS: ¿cuál crees que será el resultado de este experimento? Pue- } \\
\text { den ser una o más posibilidades. } \\
\text { (¿Qué deberías observar mientras realizas el experimento para darte } \\
\text { cuenta de los resultados?) }\end{array}$ \\
\hline MATERIALES & $\begin{array}{l}\text { ¿Qué se necesita para realizar el experimento? } \\
\text { Considera TODO: materiales descartables, elementos de laboratorio, ins- } \\
\text { trumental, material de limpieza, sustancias químicas, fósforos, etc. } \\
\text { Presenta un esquema o dibujo de los aparatos utilizados. }\end{array}$ \\
\hline METODOLOGÍA & $\begin{array}{l}\text { ¿Cómo se realiza el experimento? } \\
\text { Describe la secuencia ordenada de pasos. }\end{array}$ \\
\hline RESULTADOS & $\begin{array}{l}\text { (Al realizar la experiencia debes ir REGISTRANDO los datos de la expe- } \\
\text { riencia en función de tus HIPÓTESIS de trabajo). } \\
\text { Organizar los datos de una manera conveniente (tabla de datos, gráfica, } \\
\text { diagrama, etc.). } \\
\text { Analizar los datos e interpretarlos: ¿qué informan sobre los resultados de } \\
\text { la experiencia? }\end{array}$ \\
\hline CONCLUSIONES & $\begin{array}{l}\text { Contrastar los resultados con las hipótesis y el objetivo. ¿Los resultados } \\
\text { resuelven el problema? ¿No lo resuelven? En ambos casos explica las } \\
\text { posibles causas. }\end{array}$ \\
\hline
\end{tabular}

Tabla 1. Esquema general de informe de investigación/actividad práctica

1. Potenciar la dimensión colectiva del trabajo científico organizando equipos de trabajo y facilitando la interacción entre cada equipo y la 
comunidad científica, representada en la clase por el resto de los equipos, el cuerpo de conocimientos ya construido (recogido en los textos), el profesor como experto. Poner en debate que los resultados de una sola persona o de un solo equipo en cierta ocasión no son suficientes para modificar los modelos teóricos vigentes consensuados por la comunidad científica.

\section{El caso de los trabajos prácticos abiertos}

Algunas propuestas superadoras, en el marco del aprendizaje basado en problemas (Llorens-Molina, 2010, Ram, 1999), indican la potencialidad de los trabajos prácticos abiertos. De Jong (2011) distingue dos tipos de trabajos prácticos de acuerdo con las metas que se persigan. Cuando el objetivo del trabajo práctico es el aprendizaje de nuevas habilidades de investigación señala que se pueden proponer actividades experimentales, a las que él denomina Solución de problemas. Mientras que, si el objetivo es el aprendizaje de un nuevo conocimiento, recomienda los del tipo Planteo de problemas. La diferencia principal entre ambos es el orden en que se presentan las situaciones, como se muestra en el Esquema 2.

La propuesta para el desarrollo de trabajos prácticos de laboratorio abiertos requiere de un proceso paulatino que permita ir generando la suficiente autoconfianza en los estudiantes para participar activamente en el laboratorio, pero también en los docentes, dado que debe poder gestionar la incertidumbre del proceso y las diferentes alternativas y cuestiones que le propongan sus estudiantes. Con este fin, de Jong (2011) recomienda ir aumentando el grado de libertad (es decir, la cantidad y la calidad de las cosas que dejamos a los estudiantes para que resuelvan por su cuenta) poco a poco, paso a paso. Además, sugiere la conveniencia de organizar la clase en pequeños grupos e ir ofreciendo consejos apropiados cuando sea necesario. En todo caso, debe quedar bien claro para los alumnos el tiempo máximo disponible para realizar la actividad y otras condiciones del trabajo experimental como la limpieza, el orden o completar el cuaderno/libreta del laboratorio en donde se irán recogiendo los datos y otra información relevante. Es muy importante la supervisión permanente del docente o equipo docente, sobre todo lo que se vaya haciendo en el laboratorio. 

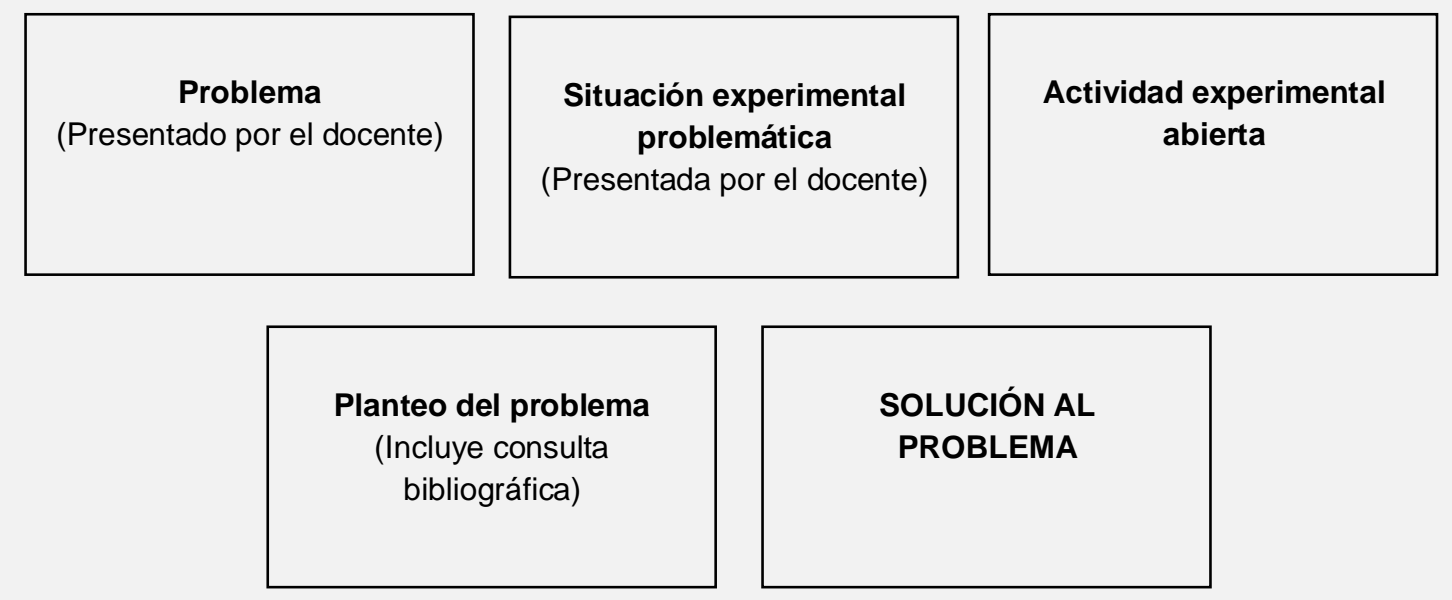

Esquema 2. Tipos de trabajos prácticos según De Jong (2011)

Para el planteo de proyectos abiertos del tipo solución de problemas, es fundamental la presencia del docente para dar consejos a los estudiantes sobre aspectos propios del diseño experimental, como, por ejemplo, los recursos disponibles, las medidas de seguridad o el tiempo que insumen ciertos procedimientos; y también para dar la retroalimentación necesaria sobre el correcto armado de equipos, la calidad de los datos recogidos, la organización de los mismos, entre otras cuestiones.

En cambio, el enfoque de los trabajos prácticos siguiendo una lógica del tipo planteo de problemas es algo diferente del anterior y suele ser menos frecuente en nuestras prácticas de enseñanza. En este caso, la situación experimental inicial es fundamental y condiciona la posibilidad de avance del trabajo práctico, por lo tanto debe ser un hecho lo suficientemente provocador como para generar la necesidad de saber más sobre ella. Si bien hay una actividad práctica inicial, generalmente mostrativa, en el diseño posterior puede haber necesidad de realizar alguna otra actividad experimental, revisar la bibliografía, aspectos que también requieren la supervisión permanente del docente durante el trabajo práctico.

\section{Para terminar}

Hasta aquí hemos revisado algunas consideraciones sobre los trabajos prácticos experimentales, en lo que se destacan dos aspectos: el primero es que la literatura discutida podría considerarse clásica, y esto se debe a que no se encuentran en la bibliografía propuestas superadoras de las aquí incluidas. El segundo es que la 
literatura suele referirse a los aspectos sobre «lo que debe ser» y son muy escasos los trabajos que presentan verdaderas transformaciones fundamentadas teóricamente. (Si bien pueden encontrarse algunas innovaciones sobre trabajos prácticos individuales, la fundamentación del cambio o la rigurosidad del análisis de los resultados obtenidos suelen quedar limitados a la experiencia concreta y a la satisfacción de sus realizadores.)

Hemos llegado entonces al momento en que cedemos el mando a los lectores para invitarlos a revisar sus trabajos prácticos experimentales. Inicialmente, para identificar en ellos los elementos que los componen, los contenidos que abarcan, los propósitos de su realización. Luego, podrán analizar los aspectos fundamentales que la justificar esta última. Por último, les proponemos el desafío de pensar sobre la posibilidad transformar algún trabajo práctico o plantear un nuevo diseño que permita aumentar el grado de libertad de los estudiantes mediante el acercamiento de las actividades de enseñanza a la naturaleza del trabajo de investigación científica.

\section{Agradecimientos}

Este trabajo pudo realizarse gracias a los subsidios obtenidos en el marco de los siguientes proyectos: ANPCYT-FONCyT PICT-2015-0044 (2016-2018), CONICET PIP № 11220130100609CO (2014-2016), UBACYT № 20020170100448BA (2018-2021), y UBANEX 10 (2018). 


\section{Referencias bibliográficas}

- Blosser, P. (1983). What research says: the role of the laboratory in science teaching, School Science and Mathematics, 83, 165-169.

- Blosser, P. (1988). Labs-Are They Really as Valuable as Teachers Think They Are? The Science Teacher, 55(5), 57-59. http://www.jstor.org/stable/24140716

- Byers, W. (2002). Promoting active learning through small group laboratory classes, University Chemistry Education, 6, 28-34.

- Calvo, I.; Zulueta, E.; Gangoiti, U. y López, J. (2008). Laboratorios remotos y virtuales en enseñanzas técnicas y científicas, Ikastorratza. e-Revista de didáctica, 3. https://dialnet.unirioja.es/servlet/articulo?codigo=2908545

- De Jong, O. (1998). Los experimentos que plantean problemas en las aulas de química: Dilemas y soluciones, Enseñanza de las Ciencias, 16(2), 305-314.

- Domin, D. (1999). A review of laboratory instruction styles, Journal of Chemical Education, 76(4), 543-547.

- Franco Moreno, R.A.; Velasco Vásquez, M.A. y Riveros Toro, C.M. (2017). Los trabajos prácticos de laboratorio en la enseñanza de las ciencias: tendencias en revistas especializadas (2012-2016). TED, 41, 37-56.

- Gil Pérez, D. y Valdés Castro (1996). La orientación de las prácticas de laboratorio como investigación: un ejemplo ilustrativo, Enseñanza de las Ciencias, 14(2), 155-163.

- Glasson, G.E. (1989). The effects of hands-on and teacher demonstration laboratory methods on science achievement in relation to reasoning ability and prior knowledge. Journal of Research in Science Teaching, 26(2), 121-131. https://doi.org/10.1002/tea.3660260204

- Gupta, V. (2001). Aims of laboratory teaching. CDTL Brief, 4(1). http://www.cdtl.nus.edu/brief/v4n1/default.htm

- Hodson, D. (1994). Hacia un enfoque más crítico del trabajo de laboratorio, Enseñanza de las Ciencias, 12(3), 299-313.

- Hofstein, A. (2017). The role of laboratory in science teaching and learning. En Taber K.S. y Akpan, B. (Eds.). Science Education: An international course companion. Sense Publishers.

- Hofstein, A. y Lunetta, V.N. (1982). The Role of the Laboratory in Science Teaching: Neglected Aspects of Research. Review of Educational Research, 52(2), 201-217. https://doi.org/10.3102/00346543052002201 
- Hofstein, A. y Mamlok-Naaman, R. (2007). The laboratory in science education: the state of the art, Chemistry Education: Research and Practice, 8(2), 105-107.

- Idoyaga, I.; Moya, N. y Lorenzo, M.G. (2017). La información en gráficos cartesianos.

Procesamiento conceptual e influencia del contenido. Enseñanza de las Ciencias, número extraordinario, 4907-4912.

- Insausti, M.J. (1997). Análisis de los trabajos prácticos de química general en un primer curso de universidad. Enseñanza de las Ciencias, 15(1), 123-130.

- Insausti, M.J. y Merino, M. (2000). Una propuesta para el aprendizaje de contenidos procedimentales en el laboratorio de física y química. Investigações em Ensino de Ciências, 5(2).

- Jalil, P. (2006). A procedural problem in Laboratory teaching: Experiment and explain, or viceversa? Journal of Chemical Education, 83(1), 159-163.

- Johnstone, A. (1993). The development of Chemistry teaching. Journal of Chemical Education, 70(9), 701-705.

- Johnstone, A.H. (2000). Teaching of chemistry - logical or psychological? Chemistry Education: Research and Practice in Europe, 1(1), 9-15.

- Johnstone, A.H. y Al-Shuaili, A. (2001). Learning in the laboratory; some thoughts from the literature. University Chemistry Education, 5, 42-51.

- Llorens-Molina, J.A. (2010). El aprendizaje basado en problemas como estrategia para el cambio metodológico en los trabajos de laboratorio, Quimica Nova, 33(4), 994-999.

- $\quad$ López Rua, A.M. y Tamayo Alzate, Ó.E. (2012). Las prácticas de laboratorio en la enseñanza de las ciencias naturales. Revista Latinoamericana de Estudios Educativos, 1(8), 145-166.

- Lorenzo, M G. y Farré, A.S. (2016). La ciencia y la tecnología entre el bien y el mal Un debate para la formación ciudadana. Aesthethika, International Journal on Subjectivity, Politics and the Arts, 12(3), 35-42. http://aesthethika.org/IMG/pdf/3340_farrelorenzo_que_es_lo_mejor_para_todos.pdf

- Lorenzo, M.G. (2008). Destilación fraccionada de ideas condensadas. Una invitación al debate sobre la naturaleza de la química. Educación en la Química, 14(1), 17-24.

- Lorenzo, M.G. (2017). Enseñar y aprender ciencias. Nuevos escenarios para la interacción entre docentes y estudiantes. Educación y Educadores, 20(2), 249-263.

- Lorenzo, M.G. (2018). Los contenidos de ciencias naturales en la enseñanza universitaria: especificidad, abstracción y orientación profesional. Aula Universitaria, (19). https://doi.org/10.14409/au.v0i19.6709 
- Lorenzo, M.G.; Reverdito, A.; Blanco, M y Salerno, A. (2012). Difficulties of undergraduate students in the organic chemistry laboratory. Problems of Education in the $21^{\text {st }}$ Century, 42(42), 74-81.

- Lorenzo, M.G.; Reverdito, A.M., Perillo, I. y Salerno, A. (2001). Los contenidos procedimentales en el laboratorio de química orgánica para la formación docente. Revista de Educación en Ciencias, 2(2), 102-105.

- Matarrita, C. y Concari, S. (2018). Características deseables en un Laboratorio Remoto para la enseñanza de la física indagando a los especialistas. Caderno Brasileiro de Ensino de Física, 35(3), 702-720. https://dialnet.unirioja.es/servlet/articulo?codigo=6816387

- Nakhleh, M.; Polles, J. y Malina, E. (2002). Learning chemistry in a laboratory environment. En Gilbert, J.K.; De Jong, O.; Justi, R.; Treagust, D. (Eds.). Chemical Education: Towards Research-based Practice (47-68). Kluger Academic Publishers.

- Ram, P. (1999). Problem-Based Learning in Undergraduate Instruction. A Sophomore Chemistry Laboratory. Journal of Chemical Education, 76(8), 1122. doi: 10.1021/ed076p1122

- Reigosa Castro, C. y Jiménez Aleixandre, M.P. (2000). La cultura científica en la resolución de problemas en el laboratorio, Enseñanza de las Ciencias, 18(2), 275-284.

- Reverdito, A. y Lorenzo, M.G. (2007). Actividades experimentales simples. Un punto de partida posible para la enseñanza de la química. Educación en la Química, 13(2), 108-121.

- Rodríguez, W. y Hernández, R. (2015). Trabajos Prácticos: una reflexión desde sus potencialidades. Góndola, Enseñanza y Aprendizaje de las Ciencias, 10(2), 15-34. doi: 10.14483/udistrital.jour.gdla.2015.v10n2.a1

- Sánchez, G.H.; Odetti, H.S.; Acuña, M.G.; Medina, G.E.; Baumann, A.J.; Marchak, G.M.; Lorenzo, M.G. (2019). Las prácticas en el laboratorio de química universitaria como escenario complejo de enseñanza. En Macedo, B.; Silveira, S.; Meziat, D.; García Astete, M. y Bengoechea, L. Enseñanza y Aprendizaje de las Ciencias en Debate, 3, 332-340. Servicio de publicaciones Universidad de Alcalá.

- Seery, M.; Agustian, H. y Zhang, X. (2018). A framework of learning in the chemistry laboratory. Israel Journal of Chemistry, 58, 1-9.

- Séré, M. (2002). La enseñanza en el laboratorio. ¿Qué podemos aprender en términos de conocimiento práctico y de actitudes hacia la ciencia? Enseñanza de las Ciencias, 20(3), 357-368.

- Talanquer, V. (2011). Macro, submicro, and symbolic: The many faces of the chemistry «triplet». International Journal of Science Education, 33(2), 179-195.

http://cbc.arizona.edu/tpp/IJSE10_Triplet.pdf 
Revisando los trabajos prácticos experimentales en

Universitaria la enseñanza universitaria | Lorenzo, María G.

- Travers, R. (Ed.) (1973). Second Handbook of Research on Teaching. Rand Mc Nally \& Co.

- Treagust, D.F.; Chittleborough, G. y Mamiala, T.L. (2003). The role of submicroscopic and symbolic representations in chemical explanations. International Journal of Science Education, 25(11), 1353-1368. 